\title{
Sonja Smets*
}

\section{THE MODES OF PHYSICAL PROPERTIES IN THE LOGICAL FOUNDATIONS OF PHYSICS}

\begin{abstract}
We present a conceptual analysis of the notions of actual physical property and potential physical property as used by theoretical physicists/mathematicians working in the domain of operational quantum logic. We investigate how these notions are being used today and what role they play in the specified field of research. In order to do so, we will give a brief introduction to this area of research and explain it as a part of the discipline known as "mathematical metascience". An in depth analysis of Aristotle's use of the notions of "actuality" and "potentiality" is presented in order to point out exactly how much of the Aristotelian connotations are embedded in the contemporary use of the concepts under investigation. Although we will not focus in depth on all the drawbacks in the early historical development of physics due to the overwhelming influence of Aristotle's writings, our analysis does touch upon some aspects of the Aristotelian theory of movement that are often overthrown nowadays.
\end{abstract}

Keywords: Actuality, Potentiality, Physical Property, Operational Quantum Logic.

*Post-doctoral Researcher at Flanders' Fund for Scientific Research. The research for this paper benefited from a bilateral scientific research project funded by the Ministry of the Flemish Community (project BIL 01/80) and the Polish State Committee for Scientific Research. I wish to thank Christian De Ronde for the many discussions on related topics and Maarten Van Dyck for his comments on the first draft of this paper. 


\section{Introduction}

Before we proceed with the main topic of our investigation namely the notions of "actuality" and "potentiality", we give a short introduction to quantum logic. In this paper we present the field of quantum logic as an important area within the discipline named "mathematical metascience" (MMS) by D. J. Foulis:

Mathematical metascience is a branch of mathematics devoted to the study of formal axiomatic systems that have been created by mathematicians, physicists, logicians, and philosophers in attempting to better understand the methods and objectives of the empirical and descriptive sciences. Such a system is admissible for study if and only if it is equipped with a heuristic or based on a paradigm that either relates directly to scientific tests, measurements or operations, or else provides a mathematical model for aspects of a scientific theory that relates, explains, and predicts the observable results or consequences of such procedures."

[13, p. 1]

We hope to convey some of the methodology of MMS in the following section while focusing on one specific branch of quantum logic. Some classifications of different branches of quantum logic are provided in $[16,19]$, while a brief history of quantum logic can be found for instance in [14]. The paper by Birkhoff and Von Neumann [7] counts as a first detailed exposition on the logical foundations of quantum physics and since its appearance, research in this domain has evolved in several directions. We will concentrate on what is known as the so-called (physical) operational approach in which the logic of experimental propositions or physical properties of quantum systems is provided with an operational dimension, i.e. a link to an experimental context. A recent overview of operational quantum logic is provided in [10]. Within this operational orientation, we narrow our view down to the Geneva approach which originated in the sixties-seventies with the work of J. M. Jauch and C. Piron $[17,18,21,22]$. The core of the Geneva approach is to provide a formal tool in order to characterize classical and quantum physical systems by means of their physical properties. How this can be achieved will be partly explained in the following section. We will first start from the notion of a (discrete) observable (or physical quantity) of a classical physical system and clarify further exactly which role the concepts of actual and potential physical properties play in the operational quantum logic of the Geneva approach. As such we have to offer a brief introduction to the theory of property lattices and focus hereby in particular on the three basic axioms which are presented 
in [25] as those axioms which seem valid for all known physical systems. Second, we concentrate in detail on Aristotle's theory of movement. We show explicitly which parts in Aristotle's use of actuality and potentiality in the case of an accidental change, can be placed in accordance with the Geneva approach's use of the notions. In the last section we trace the three basic axioms of the Geneva approach back to Aristotle. One aspect on which we focus in particular is that within the Geneva approach one views every change as the passage between what is actual and what is potential while clearly cutting this loose from the teleological connotations. In this part we will offer also an in depth analysis of Aristotle's conceptions of passing-away and comingto-be or generation and corruption. Finally, we pay special attention to the notion of an orthocomplemented property and investigate the connection to Aristotle's conceptions on contraries or opposites. In most parts of this paper we rely on our analysis presented in [26] and tend to offer a perspective on the Aristotelian concepts of actuality and potentiality and in which way their meaning changed in their usage today. In hindsight of the negative influence that Aristotle's teleological views had on the early development of physics, our conceptual analysis makes some familiar contemporary notions more accurate by placing them in the appropriate historical context.

\section{From Physical Quantities to Physical Properties}

For a recent and more detailed exposition of the basic machinery of the Geneva approach, we refer to $[8,9,20,26]$. In this section we will offer a non-technical accessible introduction and focus mainly on the notion of "physical property". Suppose we have a given classical particular physical system which is such that:

1. The given particular physical system is a characterizable and separable part of the external world.

2. The given particular physical system can be completely characterized by means if its physical properties, i.e. its two-valued physical quantities or observables.

3. The state of the given particular physical system can be represented by means of a point in a given state space, this space exists of all the points which represent the possible realizations of the given particular physical system.

In order to analyze the notion of physical property in more detail, let us denote the state space of the given system by $\Sigma$. By means of $\Sigma$, we can characterize all the systems' physical quantities. A physical quantity $X$ will 
in this picture be represented by the map $O_{X}: \Sigma \rightarrow O_{X}(\Sigma)$ which attaches to each state $\mathcal{E} \in \Sigma$ an element of another space in which $X$ is being measured. An example given by C. Piron in [22] shows that the classical physical quantity or observable "position" can be captured by the mapping from $\Sigma$ into the vector space $\mathbb{R}^{3}$. Where a physical quantity could alternatively be defined by the map which assigns to each measuring interval (value) a subset of the state space [22], a physical property corresponds to one subset of the state space. For example, the property $y$ which corresponds to the set $\mu(y)=\left\{\mathcal{E} \in \Sigma \mid O_{Y}(\mathcal{E})=1\right\}$, expresses the states in which the $\{1,0\}$-valued physical quantity $Y$ takes value 1 ; while the property $y^{\prime}$ corresponding to the set $\mu\left(y^{\prime}\right)=\left\{\mathcal{E} \in \Sigma \mid O_{Y}(\mathcal{E})=0\right\}$ expresses the states in which the $\{1,0\}$-valued physical quantity $Y$ takes value 0 . This shows that each physical quantity which takes only the two values 1 or 0 in every state of the system, is associated directly to a property (or its orthocomplement ${ }^{1}$ ) of a physical system [22]. For a given system in state $\mathcal{E} \in \Sigma$ we call the property corresponding to $\mu(y) \in P(\Sigma)$ actual if and only if $\mathcal{E} \in \mu(y)$ and potential otherwise $-P(\Sigma)$ denotes the powerset of $\Sigma$. For classical physical systems $\mu(y) \cup \mu\left(y^{\prime}\right)=\Sigma$, i.e. when a property $y$ corresponding to a twovalued observable $Y$ is potential then the orthocomplemented property $y^{\prime}$ is actual. Indeed, for classical physical systems, every property is actual or its orthocomplement is actual. For quantum physical systems, the latter utterance can not be sustained, i.e. $\mu(y) \cup \mu\left(y^{\prime}\right) \subset \Sigma$. For non-classical systems not every subset of the state space will correspond to a physical property. In this case, the union or classical disjunction of those states in which a physical property is actual and of those states in which its orthocomplement is actual does not give us the full set of states, what is lacking here are exactly the superpositions of states which have a fundamental quantum nature. On an intuitive account, following $[3,4,5,27]$, we say that when a system is in a superposition of two states (call them $s$ and $t$ ), then there exists an ideal measurement of the first kind that can force the system into state $s$ and there exists an ideal measurement of the first kind that can force the system to be in state $t^{2}$

\footnotetext{
${ }^{1}$ The notion of orthocomplementation will be more formally dealt with at the end of this section. An intuitive explanation of orthocomplementation can be given when we take a dynamic perspective. Following the dynamic approach to quantum logic recently outlined in $[3,4,5,27]$, we say that the orthocomplement of a physical property $y$ encodes the impossibility of a successful measurement of $y$.

${ }^{2}$ Details on the notion of ideal measurement of the first kind (that traces back to W. Pauli) can be found in [27].
} 
To overcome the differences in description between classical and quantum systems, the Geneva approach has introduced a new language based on the notion of yes/no question, or similarly definite experimental project. A physical property will now be specified as that what corresponds to a set of equivalent definite experimental projects. Where a definite experimental project, abbreviated as DEP, is an experimental procedure and should be thought of as a list of concrete actions. This list of actions has to be accompanied by a rule that specifies in advance when we ought to call the result at the end, positive. In order to define an equivalence relation on such a family of DEP's, the Geneva approach introduces the notion of certainty:

A given DEP is called certain (similarly the yes/no question is called true) if it is sure that the positive response would obtain should we perform the experiment [20].

If the certainty of one DEP always implies the certainty of another one, both DEP's are said to be pre-ordered. If the pre-order relation holds in both directions for two DEP's, they are called equivalent. To clarify and avoid the possible problems that the notion of "certainty" introduces in this framework (see for instance [20]), a slightly different interpretation of the basic concepts has been put forward in [11,29] and will also here be further adopted. As such performing the actions included in a DEP will be interpreted as the placing of the system in a specific (measurement) environment e. The specification of the positive result, will be interpreted as the specification of a phenomenon $\alpha$ that can occur when the system is placed in environment $e$. And further we say that a given DEP that specifies $e$ and $\alpha$, is called certain if and only if whenever the system is placed within environment $e$ then it produces phenomenon $\alpha$ to happen.

In the language provided by the Geneva approach, a physical property is called actual if and only if the DEP's which test it are certain and is potential otherwise. ${ }^{3}$ When a property is actual or not, depends on the state in which one considers the system to be. The Geneva approach adopts here a realistic stance towards physical properties. The underlying assumption is that the physical properties have an extension in reality, can be described and characterized by physicists and are considered to be measurable. In particular the EPR-"criterion of reality" (see [12]) is explicitly adopted and explains why measurability is an important ingredient. Indeed, an "actual

\footnotetext{
${ }^{3} \mathrm{~A}$ DEP which is an element of the equivalence class associated with a physical property, is conceived as a DEP which tests that property.
} 
property" is closely linked to the notion of "element of reality" introduced in $[12]$,

If, without in any way disturbing a system, we can predict with certainty $[\ldots]$ the value of a physical quantity, then there exists an element of physical reality corresponding to this physical quantity.

The Geneva approach worked out a theory of property-lattices in which the structure that holds between the properties of a classical system and quantum system differs. Several expositions of the theory of property-lattices are available, we will follow here the overview given in [29]. In order to describe the structures at stake, we first denote the set of all the physical properties of a given physical system by $\mathcal{L}$ and use the notion of "actuality" to introduce an order-relation as follows: for all $a, b \in \mathcal{L}: a \leq b$ if and only if $b$ is an actual property every time when $a$ is an actual property. The partially ordered set $\mathcal{L}$ is closed under the operation of meet: every subset $A \subseteq \mathcal{L}$ has a greatest lower bound $\bigwedge A \in \mathcal{L}$. Indeed, for every subset $A \subset \mathcal{L}$, $\bigwedge A$ is an actual property in a state $\mathcal{E}$ if and only if every $a$ in $A$ is an actual property in state $\mathcal{E}$. Operationally we call $\bigwedge A$ an actual property of a system in state $\mathcal{E}$ if it is the case that for every arbitrarily chosen environment $e_{a}$, from the collection $\left\{e_{a}\right\}_{a \in A}$, necessary to test the actuality of a property contained in $A$ we have: whenever the system is placed within an environment $e_{a}$ then it produces phenomenon $\alpha_{a}$ to happen. The use of arbitrary choice in the previous sentence is important since it indicates that a disjunction (or arbitrary choice) of environments operationally motivates the introduction of a conjunction of properties. Reversely, we cannot operationally motivate the introduction of a join of properties. Such a motivation would require a notion of simultaneity (or conjunction) of (measurement)-environments and that causes problems within quantum theory (see for instance [11]). The operational motivation for the conjunction of properties has led to many misconceptions, we refer the careful reader to $[15,28]$ for a reply to possible misunderstandings.

Since we cannot operationally introduce the join of properties, the Geneva approach applies Birkhoff's theorem for the mathematical introduction of the least upper bound, i.e. the operation called join, of any collection of properties $A$ :

$$
\bigvee A=\bigwedge\{b \in \mathcal{L} \mid \forall a \in A: a \leq b\} .
$$

Equipped with the operations of meet and join, $\mathcal{L}$ can now be called a complete lattice. It is bounded with the trivial property 1 as top element and the absurd property 0 as bottom element. Property 1 can be examined 
by means of the environment $e_{1}$ which always produces phenomenon $\alpha_{1}$ to happen whenever the system is placed within $e_{1}$, independently from its specific realization. When the system exists, property 1 will always be actual. Property 0 can be examined by means of the environment $e_{0}$ which always produces the related phenomenon $\alpha_{0}$ not to happen whenever the system is placed within $e_{0}$, independently from the specific realization of the system. Property 0 can never be "actual". It is here assumed that the environments $e_{1}$ and $e_{0}$ can always be specified for any physical system we consider.

Since the actuality of a property depends on the realization in which the system is to be considered, it is straightforward to represent states within $\mathcal{L}$. C. Piron introduced the notion of state property in [25] to refer to the strongest property of a physical system which is actual in a given realization. Formally a state property $p_{\mathcal{E} 1} \in \mathcal{L}$ is defined by:

$$
p_{\mathcal{E} 1}=\bigwedge\{a \in \mathcal{L} \mid a \text { is actual in } \mathcal{E} 1 \in \Sigma\}
$$

and given together with another state property $p_{\mathcal{E} 2}$, it satisfies the axiom:

$$
\text { If } p_{\mathcal{E} 1} \leq p_{\mathcal{E} 2} \text { then } p_{\mathcal{E} 1}=p_{\mathcal{E} 2} \text {. }
$$

This axiom states that one state property can never imply the actuality of another state property. Several properties can be actual in different states in which the system can be realized, but there is always at least one property which is actual in one state but not in another. This axiom implies also that the state properties of a physical system are the atoms in a property lattice which, following [20], can intuitively be characterized as the minimal non-zero elements.

In order to equip $\mathcal{L}$ with an orthocomplementation, one first introduces an axiom to state that for every given realization $\mathcal{E}$ there exists at least one environment $e$ which causes phenomenon $\alpha$ to happen when the system is in a possible state $\mathcal{E}^{\prime}$ which is orthogonal to $\mathcal{E}$. We conceive of the orthogonality relation on the state space as a symmetric and antireflexive separating relation. Within the context of a Kripkean style semantics for quantum logic, the notion of "accessibility" can be interpreted as "non-orthogonality". The point is that for every state property $p_{\mathcal{E}} \in \mathcal{L}$, there exists a property $p^{\sharp} \in \mathcal{L}$ which is actual in state $\mathcal{E}^{\prime}$ if and only if $\mathcal{E}$ is orthogonal to $\mathcal{E}^{\prime}$. Following [20], this allows us to represent the opposite of a property by means of the following map:

$$
{ }^{\prime}: \mathcal{L} \rightarrow \mathcal{L}: a \mapsto \bigwedge\left\{p^{\sharp} \mid p \leq a\right\} .
$$


Another axiom is needed to assure us that this map is surjective and allows us as such to say that each property is the opposite of another one. With the last axioms given, we may now call ' $: \mathcal{L} \rightarrow \mathcal{L}$ an orthocomplementation, i.e. $a \leq b \Rightarrow b^{\prime} \leq a^{\prime}, a \wedge a^{\prime}=0, a \vee a^{\prime}=1$, and $a^{\prime \prime}=a$. Within the theory of property lattices one can describe both classical and quantum systems. When the system is classical, a physical property $a \in \mathcal{L}$ or its orthocomplement $a^{\prime} \in \mathcal{L}$ is actual in every possible realization $\mathcal{E}$. When the system is quantum, a physical property and its orthocomplement can both be potential in the same realization. In general a physical system will exhibit both classical and quantum structures. Quantum logicians study these structures and focus for instance on so-called weak modular structures where the weak modular identity replaces the distributive identity and holds between the meet and join operators for specific quantum systems.

\section{Modes of Being}

The notions of actuality and potentiality can be traced back to Aristotle. We will analyze in which way Aristotle used those notions and see if it in some way matches the meaning given to them in the Geneva approach. In [1, On Generation and Corruption] and especially in [2, Metaphysics Book $\Theta$ and Book $\Delta, 7]$ Aristotle explained that there are different modes of "being", namely being potential and being actual. The transition from being potential to actual has to be placed within the context of his theory of movement and change, which is embedded with his teleological conception of causality, more precisely:

[...] everything that comes to be moves towards a principle, i.e. an end. For that for the sake of which a thing is, is its principle, and the becoming is for the sake of the end; and the actuality is the end, and it is for the sake of this that the potentiality is acquired.

[2, Metaphysics Book $\left.\Theta, 1050^{a} 7-\right]$

As is well known, Aristotle criticized Plato about his conception of ideal forms [2, Metaphysics Book I(A)]. For him, forms - shapes-were always present in the objects he considered; only in a theoretical sense may we speak of forms without objects. One of Aristotle's favorite examples is a house conceived as "bricks and timbers in such and such a position" versus a pile of bricks and timbers:

$[\ldots]$ those who define a house as stones, bricks, and timbers, are speaking of the potential house, for these are the matter; but those 
who define it as a covering for bodies and chattels, or add some other similar differentia, speak of the actuality; and those who combine both of these speak of the third kind of substance, which is composed of matter and form.

[2, Metaphysics Book H, 1043 $\left.{ }^{a} 14-\right]$

In this sense then, actuality can be interpreted as fulfillment of form while potentiality can be seen as capability of form. Aristotle put it as follows in [2, Metaphysics Book $\left.\Theta, 1050^{a} 15-\right]$ :

Further, matter exists in a potential state, just because it may attain to its form; and when it exists actually, then it is in its form.

Actuality means the existence of the thing [Metaphysics Book $\Theta, 1048^{a} 30$ ], potentiality can be assigned to non-existent things, meaning not yet actualized properties of a being, we quote:

For of non-existent things some exist potentially; but they do not exist, because they do not exist in fulfillment.

[2, Metaphysics Book $\left.\Theta, 1047^{b} 1-\right]$

Aristotle introduced potentiality and actuality to conceptualize movement and change. As such he distinguished different kinds of change, for instance change of substances is called coming-to-be, change in qualities alteration, change in magnitudes growth or diminution, and change in place locomotion. ${ }^{4}$ In [On Generation and Corruption Book I] he dealt with these different changes in detail: coming-to-be is then just the transformation of a potential substance (by no means realized) - which consists of matter and a potential form - into an actual one. This change has been called substantial change. The other kinds of change are accidental and differ from the former in the sense that they need a formal carrier - substance - for the quality, magnitude or location to be predicated of. In [On Generation and Corruption Book I] he gave several examples which explain this difference more clearly: we may think of alteration as a change from for example the musical man into the unmusical man since (un)musicalness is a property, or as Aristotle also described it, an accident of man. Without the given man, we would say that unmusicalness was a coming-to-be while musicalness a passing-away. In another example he explained growing:

\footnotetext{
${ }^{4}$ In [1, Categories, $\left.15^{a} 14-\right]$ Aristotle mentions six kinds of change, namely generation, destruction, increase, diminution, alteration, change of place, where we see generation as coming-to-be and destruction as passing-away.
} 
And as fire lays hold of the inflammable, so the active principle of growth, dwelling in the growing thing (i.e. in that which is actually flesh), lays hold of an acceding food which is potentially flesh and converts it into actual flesh. The acceding food, therefore, must be together with the growing thing; for if it were apart from it, the change would be a coming-to-be. For it is possible to produce fire by piling logs on to the already burning fire. That is growth. But when the logs themselves are set on fire, that is coming-to-be.

[1, On Generation and Corruption Book I, 322 $\left.{ }^{a} 10-\right]$

We see that things can come to be in various ways such as by addition, change of shape or by putting together as in case of a house [Physics Book I, 190 5 -]. In all these cases it is clear that "the source of becoming", as it is called in [Metaphysics Book $\Theta, 1049^{a} 12$ ], is external. As such he said that there is potentially a house if there is nothing in its matter which prevents it from becoming so in actuality. In the same way when the source of becoming is internal $1^{5}$ and those things are potentially something else, this something will become actual by itself if nothing external blocks it.

We could indeed go into further detail about the four causes Aristotle described to know the being and becoming of things, but this would lead us too far away from the subject in hand. In any case, we basically already dealt with three of the four causes Aristotle considered: (1) the material cause is the material substrate, (2) formal cause is the form to be realized, (3) the efficient cause "represents the influences from the outside world that cause the process of motion towards realization of its true nature" [30, p. 171] - cf. the above source of becoming - and (4) the final cause is then the endpoint of this process or "that for the sake of which". There still remains however one thing which, according to us, is essential to understand Aristotle's reasoning and that is his introduction of a prime or first mover. This first mover is not to be conceived of as a God who created the world but rather as the origin of all movement and change which, in itself unmovable, has to be envisaged as pure actuality. The main reason for introducing this first mover is firstly, as explained in [Metaphysics Book $\Lambda, 1071^{b} 12$-], that if there is something capable of moving things but which does not perform the act, there will be no movement at all. Secondly, if everything which moves is itself being movedcalled an intermediate mover - we end up with an infinite causal regression which according to Aristotle has to be stopped at some point [Metaphysics Book $\Lambda, 1070^{a} 3$-, Physics Book VIII, $267^{b} 1$ ]. Thirdly, eternal movement, for

\footnotetext{
${ }^{5}$ Internal means present "in the very thing which suffers change" [Metaphysics Book $\left.\Theta, 1049^{a} 13\right]$
} 
instance the rotation of planets has to be caused by an unmovable eternal substance [Metaphysics Book $\Lambda, 1073^{a} 27$ ]. We quote:

For everything that changes is something and is changed by something and into something. That by which it is changed is the primary mover; that which is changed, the matter; that into which it is changed, the form.

[Metaphysics Book $\Lambda, 1069^{b} 36-$ ]

Hereby we have captured the most essential of Aristotle's theory involving actuality and potentiality for our needs. This should allow us now to explore the "differences" of use by the Geneva approach. There are some clear points of difference right from the start: the Geneva approach does not adopt Aristotle's causal teleology - everything moves towards an end or the realization of its essence - and neither poses the existence of a first mover. The influence of Aristotle's causal teleology blocked the early development of physics, in particular it is still seen as the cause of providing wrong intuitions today. For example objects having a natural state of rest is in opposition with the law of inertia. It is not our intention to go into the details of the difficulties from which physics suffered before Galileo came around, let us stress that in the Geneva approach one is well aware of this but still finds a lot of merits in Aristotle's works. As such we can say that in a parallel way, actuality and potentiality are considered by the Geneva approach as modes of being. But we must be careful since in the latter those modes of being are only assignable to predicable properties, conceived as characteristics of physical systems. Although parallels could be established between Aristotle's notion of substance and the notion of isolated physical system, this is on the first sight not straightforward and would lead us to far away from the subject in hand. It should be clear that the Geneva approach does not introduce explicitly any notion such as "substance" or "substantial change", hence this reduces the comparison in first instance with Aristotle's use of the above mentioned notions to the cases of accidental change. Regarding actuality in the Geneva approach, an actual property is conceived as an attribute which exists; it is some realization in reality or in other words; an element of reality. Regarding potentiality on the other hand, a potential property does not exist in the same way as an actual one, it is conceived merely as a capability with respect to an actualization since there is always a chance that it could be realized after the system has been changed. The notions of actuality and potentiality are set out thus by C. Piron:

Parmi les propriétés définies pour un système donné, il y a celles que le système possède actuellement, c'est-à-dire en acte, et celles qu'il pour- 
rait acquérir, soit de lui-même, soit sous des contraintes extérieures, et qui, pour cette raison, sont dites potentielles."

[23, p. 131]

Furthermore, C. Piron sees the potential properties as those which may be actualized due to some deterministic or indeterministic change of the system. He refers explicitly to Aristotle when he explains that any such change, which involves a change of the system's state, entails a shift between its actual and potential properties:

Au cours de l'evolution, l'état change, certaines propriétés potentielles se réalisent en acte, alors que d'autres disparaissent pour n'être plus que potentielles.

$[23$, p. 132$]$

Since the main point of the Geneva approach is that every physical system can be described by means of its properties, obviously any change of a physical system can also be described by a change of its properties. It is in this sense that we have not given a direct account to Aristotle's substantial change. Also, contrary to Aristotle, change should not be taken as an action in function of some telos. In [26] we deal with special kinds of measurements and analyze deterministic and indeterministic changes a physical system can undergo, but we wish to stress here that a deterministic change is not be seen as the realization of a system's essence, and that an indeterministic change can for instance be due to any measurement which may be performed without the a priori aim of actualizing a specific potentiality. It is important that despite this, we do not give up the idea that every change can be explained as the passage between what is actual and potential and vice versa.

\section{Traces of the axioms}

In this section we refer to the above exposition of the theory of property lattices that is prevalent in the Geneva approach. The first axiom one encounters in this approach states that a state property can never imply the actuality of another state property. In [25], C. Piron refers with respect to this first axiom to Aristotle:

C'est là une loi physique déjà formulée par Aristote: si le système change, passe de l'état $\mathcal{E}_{1}$ à l'état $\mathcal{E}_{2}$, il s'enrichit de propriétés nouvelles qui s'actualisent, mais du même coup, il en perd nécessairement d'autres qui passent en potentialité, ce qui n'est pas possible si $\mathcal{E}_{1}$ est entièrement contenu dans $\mathcal{E}_{2}$.

$[25$, p. 21] 
In the context of this quote of Piron and the ones given in the previous section, we traced this physical law as C. Piron calls it, back to Aristotle's [1, On Generation and Corruption Book I, 318 ${ }^{a} 23$-; Book II, 336 23 -] where it is made clear that a "passing-away" of this is a "coming-to be" of something else, and the "coming-to be" of this is a "passing-away" of something else. In a certain sense, movement is to be conceived as becoming something and being corrupted of something else, or, when we start with something, it can be seen as obtaining some qualifications while losing others. It is in this light that Aristotle explained that there are three principles which underlie any change that takes place: form, privation and matter [2, Metaphysics Book $\Lambda$, $\left.1070^{b} 18-\right]$. As an example Aristotle dealt with colour: here the form is white, the privation is black and the matter is a surface. In [2, Metaphysics Book $\mathrm{Z}, 1033^{a} 8$-] another example is given: the matter is a man which becomes healthy - the form - when he is deprived of being invalid. So we see that becoming healthy goes hand-in-hand with the passing away of being invalid.

The next axioms which one encounters in the Geneva approach, allow us to postulate the existence of opposite properties and to ensure us that each property is the opposite of another one. Again, C. Piron in [24] finds their origin in Aristotle's work:

Un autre principe enseigné également par Aristote affirme que chaque propriété a son contraire. Ceci nous conduit à postuler que le sousensemble des états de $\Sigma$ orthogonaux à tous ceux d'un sous-ensemble représentant une propriété, représente toujours également une propriété, la propriété contraire. Enfin, dans le même ordre d'idées, nous supposerons aussi que toute propriété peut être considérée comme le contraire d'une autre.

The closest we get to this when looking at Aristotle's concept of contraries and his conception of "changement of things towards their contraries", is his saying in $\left[2\right.$, Metaphysics Book $\left.\Gamma, 1005^{a} 3-\right]$ :

For all things are either contraries or composed of contraries, and unity and plurality are the starting-points of all contraries.

In [Physics Book I, $188^{b} 25$ - $]$ this idea is extended in the way that everything that comes to be by a natural process is said to be a contrary or product of contraries. We assume that with the product of contraries Aristotle means an intermediate, derived and composed from contraries such as a colour which is derived from black and white (see [1, Physics Book I, 188 21-]). The idea is then that a thing alters "towards" contrary qualifications [1, Categories, 
$15^{b} 15$-]. With respect to the final mentioned axiom, we can even say that Aristotle in [1, Categories, $\left.11^{b} 34-\right]$ explained that a pair of contraries are said to be contraries of one another:

Things opposed as contraries, however, are never called just what they are, in relation to one another, though they are called contraries of one another. For the good is not called good of the bad, but the contrary of it; and the white not white of the black, but its contrary.

Opposites are then characterized as, among other things, contraries or as the attributes that cannot be present at the same time in that which is receptive of both [2, Metaphysics Book $\left.\Delta, 1018^{a} 20-\right]$. Note however that with Aristotle the idea is given that while two contraries cannot belong at the same moment to the same thing, it is possible that neither of them belongs when there is an intermediate, [2, Metaphysics Book I, 1056 ${ }^{a} 35$-]:

For the combined denial of opposites applies when there is an intermediate and a certain natural interval; $[\ldots]$.

We have to take into account that there is not an intermediate in all cases, for instance Aristotle gave the example of a shoe and a hand, there is nothing intermediate in that case. As such an intermediate is impossible between things that are not opposite [2, Metaphysics Book I, 1057 31-]. On the other hand, for contraries between which there is nothing intermediate, it is necessarily so that only one of them belongs to the things in which they naturally occur or are predicated of [1, Categories, $\left.12^{b} 26-\right]$. The latter case comes quite close to the "classical situation" considered by the Geneva approach in which a property or its opposite will always be actual. The fact that it is possible in non-classical systems for both a property and its opposite to be potential, in a way corresponds to the idea of an intermediate situation. Our proposition of linking Aristotle's intermediates to superposed states might well be questioned in favor of (statistical) mixed states. But we want to remark that in the outline of the Geneva approach given in this paper, we only considered so-called pure states (including superpositions) but no mixed states. Indeed, statistical mixtures are not considered part of the fundamental ingredients of the Geneva approach.

A final remark is necessary since for Aristotle it is not so that all qualifications (quality's) admit of a contrary one. Aristotle mentioned in [1, Categories, $10^{b} 15$-] that "there is no contrary to red or yellow or such colours though they are qualifications." It is on this point that we want to stress the difference with the Geneva approach where the final given axiom assures us 
that every property has an opposite one and where colours could indeed be interpreted as properties.

This paper sheds more light on the axioms of the Geneva approach and its use of the concepts of actuality and potentiality, but more work remains to be done. Indeed, other topics such as the operational principle or the notion of isolated physical system could as well be examined in a similar way. For now, we explained parts of the basic machinery of operational quantum logic and examined Aristotle's writings in order to point out some of the differences and correspondences in use of several basic concepts. We saw some striking similarities but several points of difference in the investigated topics, some clarification of which can be given by quoting C. Piron in [24]:

Mais entendons nous bien, je ne désire pas puiser dans les résultats et les conclusions de la physique d'Aristote qui sont bien sûr complètement dépassés, mais reprendre certains de ses concepts de base et adapter certains de ses arguments au problème tel que nous le connaissons aujourd'hui.

[24, p. 170]

\section{References}

[1] Aristotle, The Complete Works of Aristotle. The Revised Oxford Translation, vol. 1, J. Barnes (ed.), Princeton University Press, New Jersey (1995).

[2] Aristotle, The Complete Works of Aristotle. The Revised Oxford Translation, vol. 2, J. Barnes (ed.), Princeton University Press, New Jersey (1995).

[3] Baltag, A., and S. Smets, "The Logic of Quantum Programs", TUCS General Publication 33, 39-56, Turku Center for Computer Science (2004) arXiv: PITT-PHIL-SCI00001799.

[4] Baltag, A., and S. Smets, "Complete Axiomatizations for Quantum Actions", in Proceedings of the IQSA2004-conference, to appear in International Journal of Theoretical Physics.

[5] Baltag, A., and S. Smets, "LQP: The Dynamic Logic of Quantum Information", in Mathematical Structures in Computer Science, Special Issue on Quantum Programming Languages, submitted for publication.

[6] Baltag, A., and S. Smets, "What can Logic learn from Quantum Mechanics?", preprint to be presented at the workshop on the epistemological lessons of quantum information, ECAP05.

[7] Birkhoff, G., and J. von Neumann, "The Logic of Quantum Mechanics", Annals of Mathematics 37, 823-843 (1936), reprinted in C. A. Hooker (ed.), The Logico-algebraic Approach to Quantum Mechanics, vol. 1, D. Reidel Publishing Company, Dordrecht, pp. 1-26 (1975). 
[8] Coecke, B., D. J. Moore and S. Smets, "From Operationality to Logicality: Philosophical and Formal Preliminaries", preprint.

[9] B. Coecke, D.J. Moore and S. Smets, "From Operationality to Logicality: Syntax and Semantics", preprint.

[10] Coecke, B., D. Moore and A. Wilce, "Operational Quantum Logic: An Overview", in B. Coecke, D. Moore and A. Wilce (eds.), Current Research in Operational Quantum Logic: Algebras, Categories, Languages, Kluwer Academic Publishers, Dordrecht, pp. 1-36 (2000).

[11] Coecke, B., and S. Smets, "The Sasaki Hook is not a [Static] Implicative Connective but Induces a Backward [in Time] Dynamic One that Assigns Causes", International Journal of Theoretical Physics, to appear (arXiv: quant-ph/0111076).

[12] Einstein, A., B. Podolsky and N. Rosen, "Can Quantum-Mechanical Description of Physical Reality be Considered Complete?", Physical Review 47, 777780 (1935), reprinted in J. A. Wheeler and W. H. Zurek (eds.), Quantum Theory and Measurement, Princeton University Press, New Jersey, pp. 138-141 (1983).

[13] Foulis, D. J., "Mathematical Metascience", Journal of Natural Geometry 13, $1-50(1986)$.

[14] Foulis, D. J., "A Half-century of Quantum Logic - What Have We Learned?", in D. Aerts and J. Pykacz (eds.), Quantum Structures and the Nature of Reality, The Indigo Book of "Einstein Meets Magritte", VUB University Press/Kluwer Academic Pub., Brussels/Dordrecht (1999).

[15] Foulis, D. J., and C.H. Randall, "A Note on Misunderstandings of Piron's Axioms for Quantum Mechanics", Foundations of Physics 14, 65-81 (1984).

[16] Holdsworth, D. G., and C. A. Hooker, "A Critical Survey of Quantum Logic", Logic in the 20th Century, Scientia Special Issue, Scientia, Milan, 127-246 (1983).

[17] Jauch, J.M., Foundations of Quantum Mechanics, Addison-Wesley, Reading, Massachusetts (1968).

[18] Jauch, J. M., and C. Piron, "On the Structure of Quantal Proposition Systems", Helvetica Physica Acta 42, 842-848 (1969).

[19] Mittelstaedt, P., "Classification of Different Areas of Work Afferent to Quantum Logic", in E. G. Beltrametti and B. C. van Fraassen (eds.), Current Issues in Quantum Logic, Plenum, New York (1981).

[20] Moore, D. J., "On State Spaces and Property Lattices", Studies in History and Philosophy of Modern Physics 30, 61-83 (1999). 
[21] Piron, C., "Axiomatique quantique (PhD-Thesis)", Helvetica Physica Acta 37, 439-468 (1964). English Translation by M. Cole: "Quantum Axiomatics" RB4 Technical memo 107/106/104, GPO Engineering Department (London).

[22] Piron, C., Foundations of Quantum Physics, W. A. Benjamin Inc., Massachusetts (1976).

[23] Piron, C., "La Description d'un Système Physique et le Présupposé de la Théorie Classique", Annales de la Foundation Louis de Broglie 3, 131-152 (1978).

[24] Piron, C., "Le realisme en physique quantique: une approche selon Aristote", in E. Bitsakis (ed.), The Concept of Reality, I. Zacharopoulos, Athens (1983).

[25] Piron, C., Mécanique quantique. Bases et applications, Presses polytechniques et universitaires romandes, Lausanne (Second corrected edition 1998) First Edition (1990).

[26] Smets, S., The Logic of Physical Properties in Static and Dynamic Perspective, PhD-thesis Vrije Universiteit Brussel (2001).

[27] Smets, S., "On Causation and a Counterfactual in Quantum Logic: The Sasaki Hook", Logique et Analyse 44 (173-175), 307-325 (2001), arXiv: PITT-PHILSCI00000619.

[28] Smets, S., "In Defense of Operational Quantum Logic", Logic and Logical Philosophy 11, 191-212 (2003), arXiv: PITT-PHIL-SCI00000541.

[29] Smets, S., "From Intuitionistic Logic to Dynamic Operational Quantum Logic", Poznan Studies in Philosophy and the Humanities, to appear.

[30] Verelst, K., and B. Coecke, "Early Greek Thought and Perspectives for the Interpretation of Quantum Mechanics: Preliminaries to an Ontological Approach", in G. C. Cornelis, S. Smets, and J.P. Van Bendegem (eds.), Metadebates on Science, The Blue Book of "Einstein Meets Magritte", VUB University Press/Kluwer Academic Publishers, Brussels/Dordrecht, 163-196 (1999).

Sonja Smets

Center for Logic and Philosophy of Science

Free University of Brussels

sonsmets@vub.ac. be 